\title{
Review of: "Primary Intracranial Malignant Melanoma: A Case Report And Review Of Literature"
}

\section{Alessandro Caputo}

Potential competing interests: The author(s) declared that no potential competing interests exist.

The manuscript does not present any novel findings.

At least one histological picture would be desirable.

How did the authors exclude potential histologic mimics, e.g. melanotic schwannoma?

The authors fail to cite relevant literature on the subject. 